\title{
WASP-12b AND HAT-P-8b ARE MEMBERS OF TRIPLE STAR SYSTEMS
}

\author{
Eric B. Bechter ${ }^{1}$, Justin R. Crepp ${ }^{1}$, Henry NGo ${ }^{2}$, Heather A. Knutson ${ }^{2}$, Konstantin Batygin $^{2}$, \\ Sasha Hinkley ${ }^{3,7}$, Philip S. Muirhead ${ }^{3,4,8}$, John Asher Johnson ${ }^{2,5}$, Andrew W. Howard ${ }^{6}$, \\ Benjamin T. Montet ${ }^{3,9}$, Christopher T. Matthews ${ }^{1}$, and Timothy D. Morton ${ }^{3}$ \\ ${ }^{1}$ Department of Physics, University of Notre Dame, 225 Nieuwland Science Hall, Notre Dame, IN 46556, USA; ebechter@nd.edu \\ ${ }^{2}$ Department of Planetary Science, California Institute of Technology, 1200 East California Boulevard, Pasadena, CA 91125, USA \\ ${ }^{3}$ Department of Astronomy, California Institute of Technology, 1200 East California Boulevard, Pasadena, CA 91125, USA \\ ${ }^{4}$ Institute for Astrophysical Research, Boston University, 725 Commonwealth Avenue, Boston, MA 02215, USA \\ ${ }^{5}$ Harvard-Smithsonian Center for Astrophysics, Cambridge, MA 02138, USA \\ ${ }^{6}$ Institute for Astronomy, University of Hawaii, 2680 Woodlawn Drive, Honolulu, HI 96822, USA \\ Received 2013 July 25; accepted 2014 April 17; published 2014 May 16
}

\begin{abstract}
We present high spatial resolution images that demonstrate that WASP- $12 \mathrm{~b}$ and HAT-P-8b orbit the primary stars of hierarchical triple star systems. In each case, two distant companions with colors and brightnesses consistent with $\mathrm{M}$ dwarfs co-orbit the hot Jupiter planet host as well as one another. Our adaptive optics images spatially resolve the secondary around WASP-12, previously identified by Bergfors et al. and Crossfield et al. into two distinct sources separated by $84.3 \pm 0.6$ mas $(21 \pm 3 \mathrm{AU})$. We find that the secondary to HAT-P-8, also identified by Bergfors et al., is in fact composed of two stars separated by $65.3 \pm 0.5$ mas $(15 \pm 1 \mathrm{AU})$. Our follow-up observations demonstrate physical association through common proper motion. HAT-P-8 C has a particularly low mass, which we estimate to be $0.18 \pm 0.02 M_{\odot}$ using photometry. Due to their hierarchy, WASP-12 BC and HAT-P-8 BC will enable the first dynamical mass determination for hot Jupiter stellar companions. These previously well studied planet hosts now represent higher-order multi-star systems with potentially complex dynamics, underscoring the importance of diffraction-limited imaging and providing additional context for understanding the migrant population of transiting hot Jupiters.
\end{abstract}

Key words: astrometry - stars: individual (WASP-12, HAT-P-8) - techniques: high angular resolution techniques: photometric

Online-only material: color figure

\section{INTRODUCTION}

There is much debate regarding the origin and evolutionary history of hot Jupiters. Traditional core accretion theory suggests that such planets form beyond the ice-line (the boundary outside which water exists in a frozen state) prior to moving inward (Pollack et al. 1996). The earliest proposed planet migration mechanisms involve a gradual inward-spiral facilitated by planet-disk interactions (Goldreich \& Tremaine 1980; Lin et al. 1996; Murray et al. 1998). Naive interpretation of these migration models presumes that planetary orbits should be well aligned with the spin-axes of their host stars. However, precision radial velocity $(\mathrm{RV})$ measurements exploiting the Rossiter-Mclaughlin (RM) effect show that many transiting hot Jupiter orbits are significantly misaligned (Winn et al. 2009, 2010b; Triaud et al. 2010; Hébrard et al. 2011; Albrecht et al. 2012).

Numerous dynamical models have been proposed to explain the wide range of observed spin-orbit angles, including planet-planet scattering (Ford \& Rasio 2008; Chatterjee et al. 2008) and Kozai-Lidov perturbations with subsequent tidal friction (Wu \& Murray 2003; Naoz et al. 2011). Several teams have performed comparative analyses suggesting that these two modes could be responsible for placing Jupiters into very short (several day) orbital periods, either individually or in combination (Fabrycky \& Winn 2009; Morton \& Johnson 2011;

\footnotetext{
7 NSF Postdoctoral Fellow.

8 Hubble Fellow.

9 NSF Graduate Research Fellow.
}

Nagasawa \& Ida 2011; Beaugé \& Nesvorný 2012; Dawson \& Murray-Clay 2013).

The underlying assumption motivating these more complex dynamical models is that protoplanetary disks maintain alignment with their host stars throughout the planet formation process. However, this assertion need not apply to all stars. Recent theoretical work indicates that the cause of misalignment may instead be induced by forces acting on the disk itself. For example, Lai et al. (2011) have proposed that young protostars with strong magnetic fields $\left(>10^{3} \mathrm{G}\right)$ can act to warp and misalign the circumstellar disk. Alternatively, gravitational torques from a companion star can change the inclination of the disk relative to the spin-axis of the star prior to the formation of planets (Batygin 2012). In any case, such mechanisms must be able to account for the observed abrupt change in the distribution of spin-axis angles as a function of stellar effective temperature (Winn et al. 2010a; Albrecht et al. 2012).

A number of plausible hot Jupiter migration mechanisms involve the presence of a massive third body. High spatial resolution imaging can detect such companions at physical scales corresponding to the expected location of their orbits (Eggenberger et al. 2007; Daemgen et al. 2009; Mugrauer \& Neuhäuser 2009; Mason et al. 2011; Roberts et al. 2011; Ginski et al. 2012; Faedi et al. 2013; Narita et al. 2012). ${ }^{10}$ These studies consistently find that a significant fraction (tens of percent) host a distant stellar candidate companion that could potentially affect the dynamical histories of the observed hot

\footnotetext{
10 Stellar companions at short orbital periods can be constrained and sometimes ruled out by existing RV measurements.
} 
Table 1

Summary of Astrometric Measurements Listing Integration Time ( $\left.\Delta t_{\text {int }}\right)$, Angular Separation $(\rho)$, and Position Angle (P.A.)

\begin{tabular}{|c|c|c|c|c|c|c|c|}
\hline \multirow[t]{2}{*}{ Companion } & \multirow[t]{2}{*}{ JD-2,450,000 } & \multirow{2}{*}{$\begin{array}{l}\text { Date } \\
\text { (UT) }\end{array}$} & \multicolumn{3}{|c|}{$\Delta t_{\text {int }}(\mathrm{s})$} & \multirow{2}{*}{$\begin{array}{c}\rho \\
(\mathrm{mas})\end{array}$} & \multirow{2}{*}{$\begin{array}{l}\text { P.A. } \\
\left(^{\circ}\right)\end{array}$} \\
\hline & & & $J$ & $K^{\prime}$ & $K_{s}$ & & \\
\hline WASP-12 B & $5,959.9$ & 2012 Feb 2 & 135 & 135 & & $1064 \pm 19$ & $251.3 \pm 1.0$ \\
\hline WASP-12 C & $\ldots$ & $\ldots$ & $\ldots$ & $\ldots$ & & $1073 \pm 19$ & $246.8 \pm 1.0$ \\
\hline WASP-12 B & $6,353.8$ & 2013 Mar 2 & & & 150 & $1062 \pm 18$ & $251.4 \pm 1.0$ \\
\hline WASP-12 C & $\cdots$ & $\cdots$ & & & $\cdots$ & $1072 \pm 18$ & $247.1 \pm 1.0$ \\
\hline HAT-P-8 B & $6,103.0$ & 2012 Jun 24 & 162 & 95 & & $1040 \pm 14$ & $137.9 \pm 0.8$ \\
\hline HAT-P-8 C & $\ldots$ & $\ldots$ & .. & $\ldots$ & & $1049 \pm 14$ & $141.4 \pm 0.8$ \\
\hline HAT-P-8 B & $6,476.9$ & 2013 Jul 3 & 180 & & 180 & $1053 \pm 14$ & $137.6 \pm 0.8$ \\
\hline HAT-P-8 C & $\ldots$ & $\ldots$ & $\ldots$ & & $\ldots$ & $1041 \pm 14$ & $140.7 \pm 0.8$ \\
\hline
\end{tabular}

Note. Observations are separated by more than $1 \mathrm{yr}$ for each stellar system.

Jupiters. Several of the most comprehensive and recent programs have used "lucky" imaging to efficiently explore a large number of targets. However, near-infrared observations combined with adaptive optics (AO) provide comparatively deeper effective contrast levels especially for objects with red colors such as M dwarfs and brown dwarfs (Fleming et al. 2012). We have recently commenced a multi-faceted observing program, named "Friends of Hot Jupiters" (hereafter FHJ), that systematically searches for additional companions around a large sample of transiting planet systems (Knutson et al. 2014). The primary objective of the FHJ survey is to quantify the relative fraction of systems including both well aligned and misaligned hot Jupiters that contain distant tertiary bodies and to study any candidate perturbers in detail using imaging and spectroscopy.

In this paper, we present initial results from the FHJ survey demonstrating that WASP-12 and HAT-P-8 are actually triple star systems. The candidate companion pairs found orbiting these two planet hosts were identified previously by Bergfors et al. (2011) as single objects. Our diffraction limited observations using Keck spatially resolve each secondary source into two distinct components. Combining our measurements with previous observations increases the astrometric time baseline by a factor of two to three and allows us to confirm the physical association of these objects with their parent stars.

\section{SUMMARY OF PREVIOUS OBSERVATIONS}

\subsection{WASP -12}

WASP-12b is a highly irradiated transiting hot Jupiter that orbits a G0V star with a 1.09 day period (Hebb et al. 2009). RM measurements yield a sky-projected spin orbit angle of $\lambda=59_{-20}^{+15}$ deg (Albrecht et al. 2012). WASP-12b may have a prolate shape and be undergoing Roche-Lobe overflow that results in substantive mass loss ( $\mathrm{Li}$ et al. 2010; Fossati et al. 2010, 2013). It has been suggested that this planet's dayside emission spectrum is consistent with a super-solar carbon-tooxygen ratio (Madhusudhan et al. 2011; Moses et al. 2013; see, however, Crossfield et al. 2012). Recent observations of WASP$12 \mathrm{~b}$ 's transmission spectrum indicate that it may also have a high-altitude haze or cloud layer (Swain et al. 2013; Stevenson et al. 2013).

Bergfors et al. (2011) detected a faint source separated by $1.047 \pm 0.021$ from the WASP-12 primary. Using Keck/ NIRSPEC archival data, Crossfield et al. (2012) analyzed the near-infrared spectrum of the candidate companion and found that that it is consistent with an M dwarf. Crossfield et al. (2012) also note that the candidate is abnormally bright for an M dwarf if situated at the same distance as the primary. Bergfors et al. (2013) find that the companion's point-spread function (PSF) appears to be elongated in two separate epochs, possibly indicating that it is a marginally resolved triple system.

\subsection{HAT-P-8}

HAT-P-8b is a transiting hot Jupiter that orbits an F5V star with a period of 3.07 days (Latham et al. 2009). Initially suspected to have an inflated radius, recent observations by Mancini et al. (2013) indicate a higher density than previously reported. Simpson et al. (2011) measure a sky-projected spinorbit angle of $\lambda=15_{-43}^{+33} \mathrm{deg}$ and Moutou et al. (2011) find $\lambda=-17_{-11.5}^{+9.2} \mathrm{deg}$, both consistent with a reasonably well aligned prograde orbit. High spatial resolution imaging by Bergfors et al. $(2011,2013)$ indicates that HAT-P-8 may be part of a binary star system although Faedi et al. (2013) were unable to confirm the candidate companion, which had a purported angular separation of $1^{\prime \prime} .027 \pm 0^{\prime \prime} 011$.

\section{ADAPTIVE OPTICS IMAGING}

We initially observed WASP-12 $(V=11.6)$ and HAT-P-8 $(V=$ 10.4) as part of the FHJ program in Spring 2012 using NIRC2 (instrument PI: Keith Matthews) with the Keck II AO system (Wizinowich 2000). Our standard procedure for searching the immediate vicinity of transiting planet hosts involves executing a three-point dither pattern that facilitates removal of instrument and sky background radiation while avoiding the (noisy) bottom left quadrant of the NIRC2 array. Observations are nominally obtained in position angle mode without allowing for field rotation since we do not perform PSF subtraction. We used the NIRC2 narrow camera setting to provide fine (10 mas) spatial sampling of the instrument PSF. Integration times for all observations are listed in Table 1.

The data were processed using standard techniques to flatfield the array, replace hot pixels, subtract background noise, and align and co-add the frames (e.g., Crepp et al. 2012). Figure 1 shows the final reduced $K$-band images for WASP-12 and HAT$\mathrm{P}-8$. Our observations provide a spatial resolution comparable to the diffraction limit (approximately 45 mas). In each case, two candidate companions $(\mathrm{B}, \mathrm{C})$ are detected.

We obtained complementary photometry in the $J$ band to determine the companion colors and help constrain their physical properties. WASP-12 BC are spatially resolved in the $J$ band; however, HAT-P-8 BC are not seen in the UT 2012 June 24 $J$-band images due to high airmass (2.19) indicating that the HAT-P-8 companions have red colors. Deeper follow-up J-band 

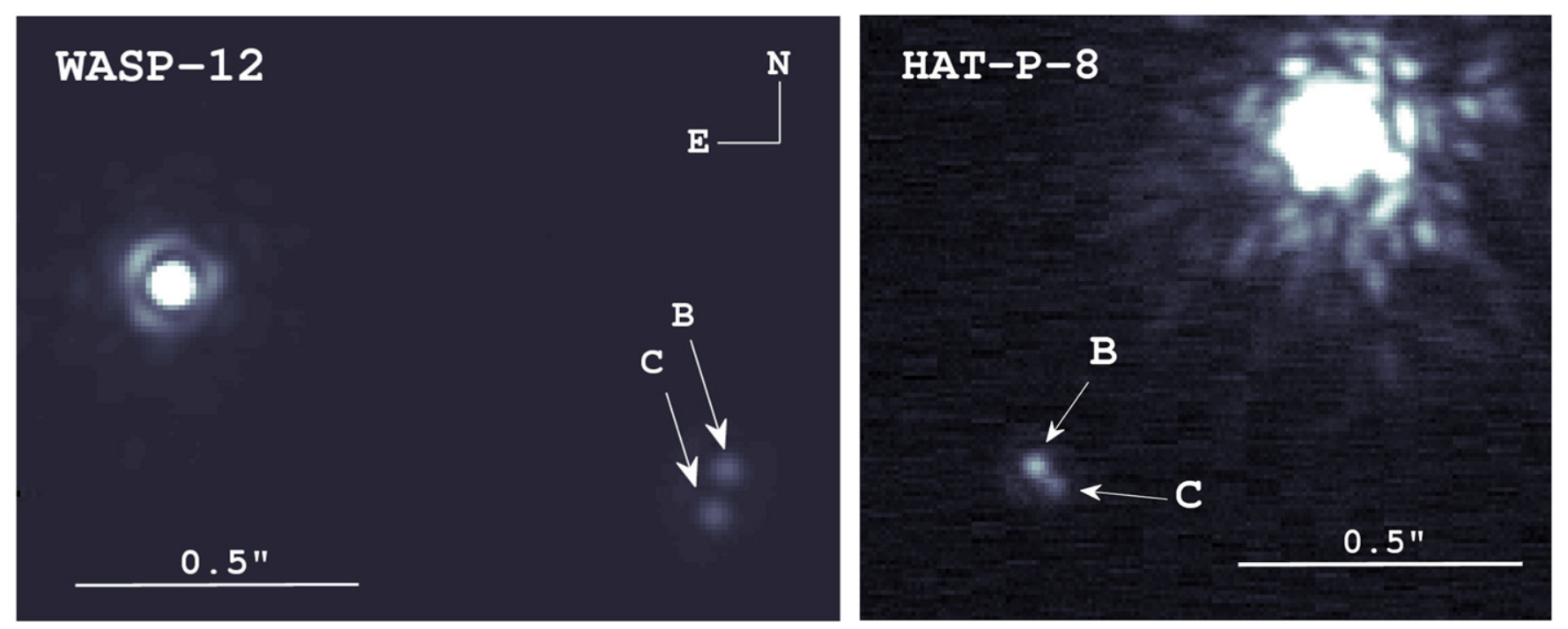

Figure 1. Keck AO discovery images of WASP-12 B,C taken on UT 2012 February 2 (left) and HAT-P-8 B,C taken on UT 2012 June 24 (right). North is up and east is left in both images. Follow-up observations separated by more than 1 yr recover each companion.

(A color version of this figure is available in the online journal.)

observations taken UT 2013 July 3 (see Section 4.2) detect the combined light of HAT-P-8 BC but do not spatially separate the objects as is seen at longer wavelengths.

\section{PHOTOMETRY AND ASTROMETRY}

\subsection{PSF Model Fits}

We perform a Bayesian analysis to model the AO observations of WASP-12 and HAT-P-8 at each epoch. Specifically, Markov Chain Monte Carlo (MCMC) numerical methods are employed to compute companion relative brightnesses, astrometric positions, and determine uncertainties. The Metropolis-Hastings algorithm efficiently explores regions of parameter space to find the best-fitting global minimum and calculate posterior distributions for each fit parameter.

We simultaneously model three PSFs to self-consistently account for contamination from a nearby companion star. Free parameters include: rectilinear coordinates for each source; peak brightness of each source; sky background levels (which we model as spatially uniform); and PSF fitting parameters, $\alpha, \beta$, $\gamma, r_{s}$, and $w$. The observations are modeled using a modified Moffat function given by:

$$
I(x, y)=\sum_{i=1}^{i=3}\left\{\alpha_{i}\left[1+\left(\frac{r_{i}}{r_{s}}\right)^{2}\right]^{-\beta}+\gamma_{i} e^{-r_{i}^{2} / w^{2}}\right\},
$$

where $r_{i}=\sqrt{\left(x_{i}-x_{0 i}\right)^{2}+\left(y_{i}-y_{0 i}\right)^{2}}$ is a polar coordinate corresponding to the angular separation from each source, $i$, in the image. The term on the left describes the AO halo and the term on the right characterizes the PSF core. By separating the terms, we effectively account for tip/tilt and focal anisoplanatism in the images, although we do not allow $r_{s}$ and $w$ to vary individually $\left(w_{i}=w, r_{s_{i}}=r\right)$ due to the already large number of degrees of freedom (12 when including the sky background). The posterior distributions found by our MCMC algorithm marginalize over all fitting parameters.

Equation (1) captures on-axis AO features but does not replicate low order aberrations or diffraction from the first Airy ring. We have experimented with other PSF forms such as $\operatorname{sinc}(\ldots)$ and $\operatorname{sinc}^{2}(\ldots)$ functions. Assuming that uncertainties in each reduced image are described by Poisson statistics at the pixel level, resulting from sky-background subtraction shot noise, we find the results from each $\mathrm{AO}$ model are consistent with one another but uncertainties are unrealistically small. For example, angular separation measurement uncertainties are less than 1 mas $(1 \sigma)$. The images used for our analysis have been fully processed prior to MCMC calculations. As such, we have stacked frames acquired from different dither positions. This step is required because the companions are so much fainter than their primary star, particularly in the $J$ band. However, by combining images obtained from different locations on the array, we have introduced PSF spatial smearing from uncorrected optical distortions. We estimate the size of this effect using polynomial fits available for the NIRC2 array provided by Keck Observatory. ${ }^{11}$ Systematic errors are of order 1-2 pixels and change depending on the size of the dither pattern. Distortion corrections may be applied before image stacking but this can introduce significant numerical noise. Furthermore, the correction coefficients also change slowly with time (Yelda et al. 2010).

Our final adopted astrometric uncertainties were found by adding the effects of optical distortion in quadrature with that from photon noise and pixel crosstalk resulting from PSF fitting errors. We self-consistently account for uncertainty in the plate scale and orientation of the NIRC2 array (Ghez et al. 2008) by randomly drawing values for the plate-scale and orientation from a normal distribution and folding the results into calculations of the angular separation and position angle when converting from pixel separations to arcseconds. The effect from optical distortion dominates the uncertainty for each astrometric epoch as it is much greater than both cross-talk and photon noise. Results for relative astrometry measurements are shown in Table 1. Although our observations from 2012 and 2013 were acquired in different filters $\left(K^{\prime}\right.$ and $\left.K_{S}\right)$, due to a change in the FHJ default observing strategy, this effect appears to be small since the results are nearly identical.

\footnotetext{
11 Distortion correction polynomials found at http://www2.keck.hawaii.edu/inst/nirc2/dewarp.html.
} 

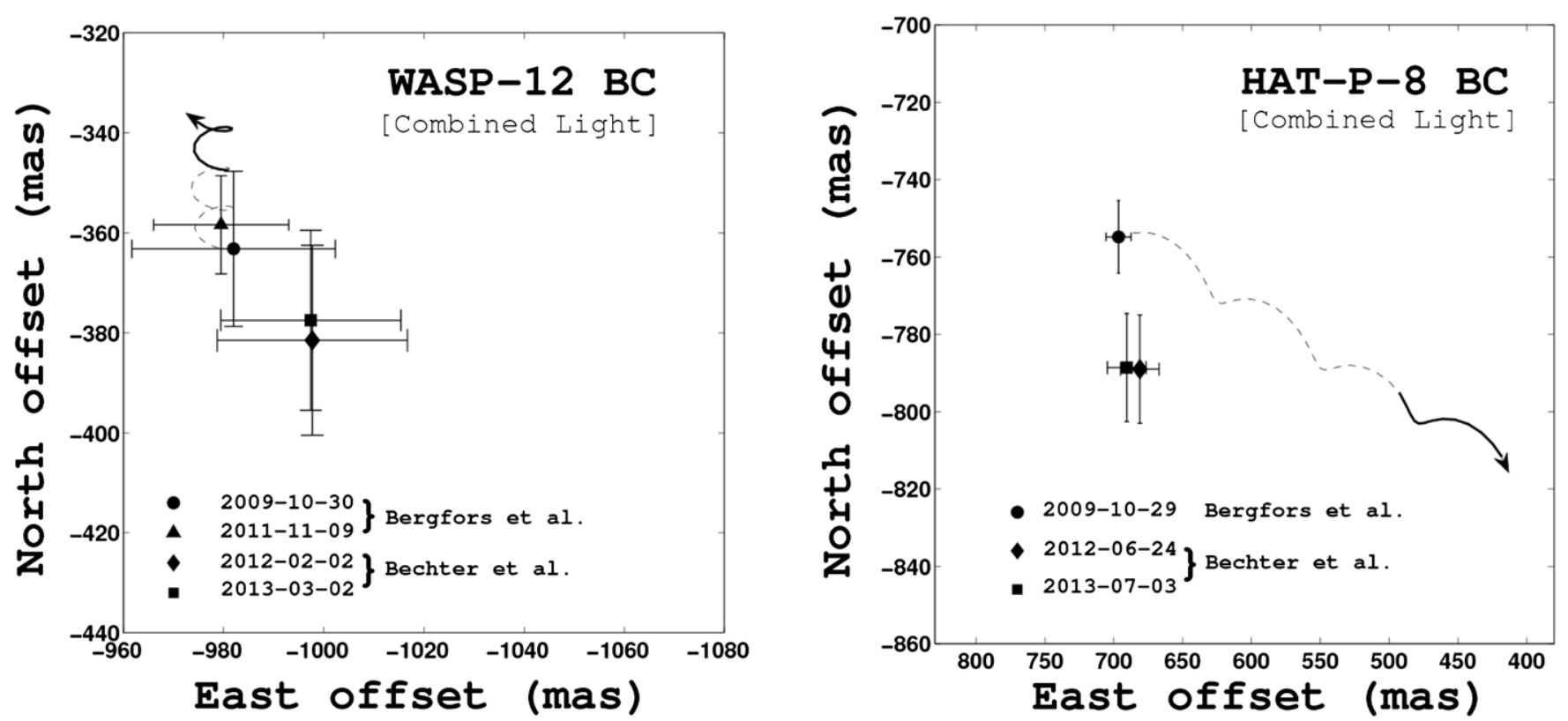

Figure 2. Astrometric measurements for WASP-12 and HAT-P-8. Axes correspond to the angular separation (offset) in the north and east cardinal directions as measured relative to the primary star. The combined proper motion plus parallactic motion of an infinitely distant (unassociated) object is given by the dashed and solid curves. Dashed curves correspond to the astrometric time baseline of Bergfors et al. (2013). The solid curves correspond to the astrometric time baseline of this study. Bergfors et al. (2013) did not spatially resolve the WASP-12 BC and HAT-P-8 BC components, but did provide the initial detection of their combined light signal (in 2009 October). We plot the photo-center of our resolved BC companions to compare to Bergfors' 2009 and 2011 data. Our Keck AO epochs are separated by more than $1 \mathrm{yr}$ and demonstrate physical association (by themselves) for HAT-P-8; association of WASP-12 BC is established by combining our results with those of Bergfors et al. (2013). Our astrometric uncertainties are over-plotted and comparable to Bergfors et al. (2013). Our measurement precision is dominated by systematics from distortion in the individual frames. Orbital motion of these two bodies may be detectable with additional observations.

\subsection{Physical Association}

We perform an astrometric analysis to assess the physical association of the WASP-12 BC and HAT-P-8 BC candidates with their primary star. To do so, we compare our astrometric measurements against the null hypothesis that the off-axis sources are infinitely distant unrelated background objects with zero parallax. WASP-12 has a small proper motion $\left[-0.7,-7.8\right.$ mas $\left.\mathrm{yr}^{-1}\right]$ comparable to the size $(9.963 \pm$ 0.006 mas) of a NIRC2 pixel (Høg et al. 2000; Ghez et al. 2008). HAT-P-8 has a proper motion that is an order of magnitude larger [75.5, 17.5 mas yr $\left.^{-1}\right]$ (Høg et al. 2000). Neither star has a Hipparcos parallax measurement, which complicates the analysis. Instead, the distance to WASP-12 is estimated using a photometric distance modulus (Bergfors et al. 2013) and the distance to HAT-P-8 is determined using a spectroscopic distance modulus (Latham et al. 2009). We incorporate parallactic motion by converting estimated distances to a trigonometric parallax (ellipse). The resulting differential motion across the sky between the primary star and candidate secondary/tertiary is given by the vector sum of the proper and parallactic motion (Zimmerman et al. 2010).

Our astrometric measurements are shown in Figure 2. Overplotted are previous measurements taken by Bergfors et al. (2013) in 2009 October that identify the combined light signal of WASP-12 BC and HAT-P-8 BC but do not spatially resolve the sources into individual components. Our Keck AO observations from 2012 and 2013 clearly separate the light from each companion star. The angular separation of WASP-12 BC is $84.3 \pm 0.6$ mas $(21 \pm 3 \mathrm{AU})$ and the angular separation of HAT-P-8 BC is only $65.3 \pm 0.5$ mas $(15 \pm 1 \mathrm{AU})$, comparable to the diffraction limit of a $10 \mathrm{~m}$ telescope at near-infrared wavelengths (Figure 1). Optical distortion for such small separations is negligible. To compare data on an equal footing with
Bergfors et al. (2013), we plot combined light photo-centers for WASP-12 BC and HAT-P-8 BC in Figure 2.

The a priori probability of finding three point sources in a hierarchical configuration separated by only $1^{\prime \prime}$ on the sky is very low. Our two astrometric epochs for WASP-12 and HATP-8 are separated by 393.9 days and 373.9 days, respectively. The expected motion of a background source relative to the primary star is $8.5 \pm 1.0$ mas $(0.9 \pm 0.1$ pixels $)$ for WASP-12 and $79.3 \pm 2.9$ mas $(8.0 \pm 0.3$ pixels $)$ for HAT-P-8 over the same time-frame.

With only two observations, the confirmation that WASP-12 $\mathrm{BC}$ are bona-fide companions is marginal. However, combining our measurements with the 2009 October initial detection from Bergfors et al. (2011), we can demonstrate that the three point sources are physically associated (Figure 2). To further reinforce our results, we have determined the photometric distance modulus for WASP-12 BC. The combined light apparent magnitude of the WASP-12 System is $10.19 \pm 0.02$ (Skrutskie et al. 2006). Backing out the individual apparent magnitudes of WASP-12 BC from our relative photometry measurements, we find the distance to WASP-12 B is $263 \pm 13 \mathrm{pc}$ and the distance to WASP-12 C is $267 \pm 13 \mathrm{pc}$. These values overlap with the photometric distance estimated by Bergfors et al. (2013) of $250 \pm 30 \mathrm{pc}$, effectively ruling out the possibility that they are foreground or background objects.

HAT-P-8 BC are confirmed using our observations alone due to the large space motion of the host star. We cannot claim detection of orbital motion for either system because of the aforementioned systematic errors and the fact that the stars were observed with different instruments and filters. Dedicated astrometric measurements are required to determine the total dynamical mass of the secondary and tertiary in each case (Dupuy et al. 2010). We note that in both cases, WASP-12 and HAT-P-8, our measurements are $\approx 20$ mas south and $\approx 20$ mas 

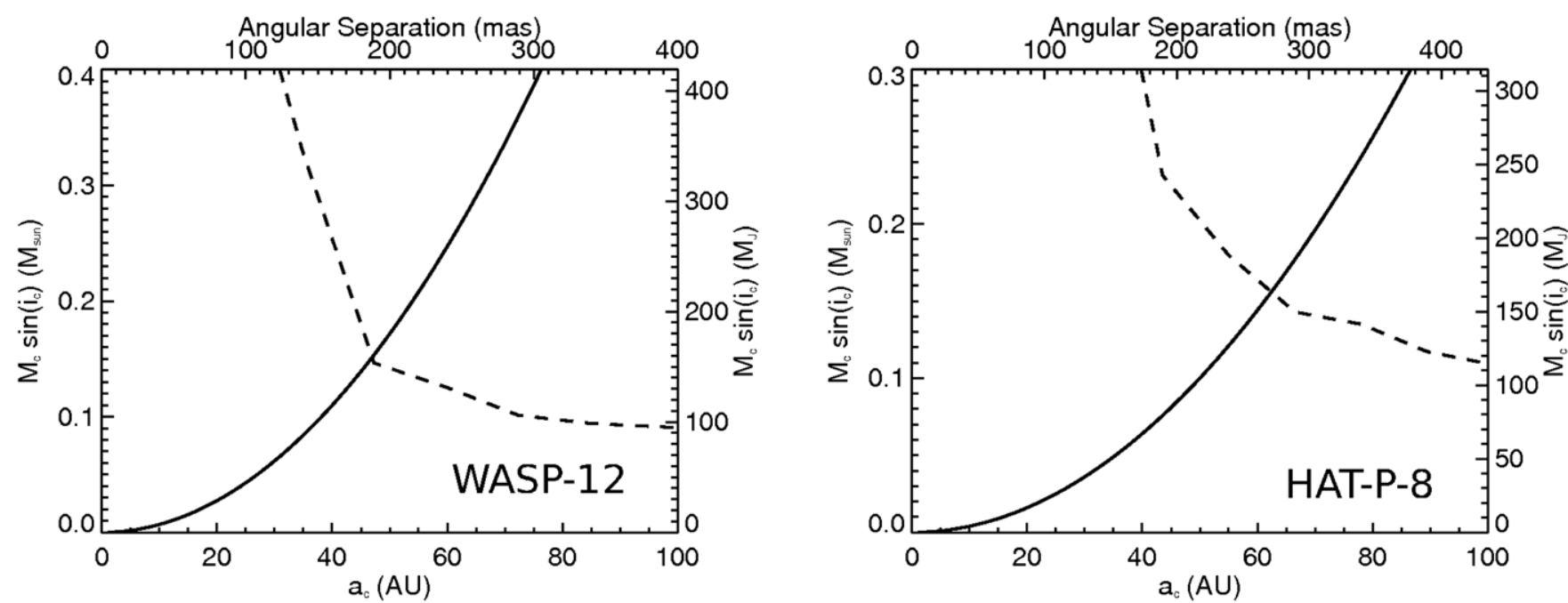

Figure 3. Joint RV and imaging constraints on the presence of additional companions orbiting WASP-12 (left) and HAT-P-8 (right) using the accelerations listed in Equation (2). Any unseen stars, brown dwarfs, or gas giant planets must lie below both the limits set by Doppler RV measurements (solid line) and those set by AO imaging (dashed line).

Table 2

Secondary and Tertiary Companion Photometric Properties

\begin{tabular}{lcccccc}
\hline \hline Companion & $\Delta J$ & $\Delta K_{S}$ & $J-K_{s}$ & $M_{K_{s}}$ & $\begin{array}{c}\text { Mass } \\
\left(M_{\odot}\right)\end{array}$ & Spec. Type \\
\hline WASP-12 B & $3.81 \pm 0.05$ & $3.25 \pm 0.04$ & $0.85 \pm 0.08$ & $6.47 \pm 0.27$ & $0.38 \pm 0.05$ & M3V \\
WASP-12 C & $3.92 \pm 0.05$ & $3.28 \pm 0.04$ & $0.93 \pm 0.08$ & $6.50 \pm 0.27$ & $0.37 \pm 0.05$ & M3V \\
\hline HAT-P-8 B & $\ldots$ & $5.58 \pm 0.07$ & $\ldots$ & $7.73 \pm 0.16$ & $0.22 \pm 0.03$ & $\approx \mathrm{M} 5 \mathrm{~V}$ \\
HAT-P-8 C & $\cdots$ & $6.08 \pm 0.10$ & $\ldots$ & $8.32 \pm 0.17$ & $0.18 \pm 0.02$ & $\approx \mathrm{M} 6 \mathrm{~V}$ \\
\hline
\end{tabular}

Notes. We estimate spectral types using near-infrared color information (when available) and absolute magnitudes by comparing to Kraus \& Hillenbrand 2007. Absolute magnitudes are found using (photometric or spectroscopic) distance modulus estimates: $d=250 \pm 30 \mathrm{pc}$ for WASP-12 (Bergfors et al. 2013) and $d=230 \pm 15$ pc for HAT-P-8 (Latham et al. 2009).

east of Bergfors, suggesting possible systematics between the AstraLux and Keck data sets.

\subsection{Companion Characterization}

Bergfors et al. (2013) assign a preliminary spectral type of M0V for WASP-12 "B" (combined light), assuming the identified off-axis source is associated. Crossfield et al. (2012) find that WASP-12 "B" is a hot $\mathrm{M}$ dwarf with $\Delta K=$ $2.45 \pm 0.06 \mathrm{mag}$. We find that WASP-12 B and WASP-12 C are $\Delta K_{s}^{A, B}=3.25 \pm 0.04$ and $\Delta K_{s}^{A, C}=3.28 \pm 0.04 \mathrm{mag}$ fainter than the primary, respectively (Table 2). Combining the signal from both components, our measurements show that the expected unresolved brightness difference between the secondary/tertiary and primary star should be $\Delta K_{s}^{A, B C}=$ $2.51 \pm 0.03 \mathrm{mag}$, consistent with the interpretation of Crossfield et al. (2012).

To further characterize the companions around each star, we calculate absolute magnitudes based on previous distance estimates from Bergfors et al. (2013) and Latham et al. (2009). Our uncertainty in absolute magnitude is dominated by the lack of a trigonometric parallax measurement. We estimate the mass of each companion using Girardi et al. (2002) evolutionary models assuming a system age of 5 Gyr. Comparing our absolute magnitudes to those of Kraus \& Hillenbrand (2007), we find that WASP-12 BC are consistent with M3V (Table 2). Additionally, the $J-K$ colors of WASP-12 BC (see Table 2) are also consistent with those of $\mathrm{M}$ stars (Kraus \& Hillenbrand 2007). Although HAT-P-8 BC are detected during second epoch
(UT 2013 July 3) observations, they are spatially unresolved in the $J$ band because the images were obtained at an airmass of 2.19. Performing aperture photometry for the pair, we find a combined difference in magnitude of $\triangle J^{A, B C} J=5.9 \pm 0.2$. We estimate the spectral types of HAT-P-8 B and C to be $\approx \mathrm{M} 5 \mathrm{~V}$ and $\approx \mathrm{M} 6 \mathrm{~V}$, respectively, using $K$-band photometry alone.

\subsection{Companion Constraints}

As part of the FHJ program, we obtained additional RV measurements for both systems, which we use to constrain the presence of additional companions at shorter orbital periods. Our best-fit RV slopes are:

$$
\begin{aligned}
d v / d t_{\mathrm{WASP}-12} & =-4.12 \pm 4.37 \mathrm{~m} \mathrm{~s}^{-1} \mathrm{yr}^{-1} \\
d v / d t_{\mathrm{HAT}-P-8} & =-2.72 \pm 2.39 \mathrm{~m} \mathrm{~s}^{-1} \mathrm{yr}^{-1}
\end{aligned}
$$

consistent with the absence of massive, $m \geqslant 5 M_{J}$, objects out to $a \leqslant 8.3$ AU for WASP-12 and $a \leqslant 10.9$ AU for HAT-P-8. Figure 3 displays joint constraints imposed by the combination of Doppler RV measurements (solid lines) and direct imaging observations (dashed lines). Should any additional companions be present in these systems, their masses must reside below both curves. Continued RV monitoring of the host stars will further eliminate regions of mass-semi-major-axis parameter space.

\section{SUMMARY AND DISCUSSION}

We have commenced a multi-disciplinary follow-up observing program, named "Friends of Hot Jupiters" (FHJ), that 
targets a large sample of short-period gas giant transiting planet systems. In this paper, we present AO images from Keck that spatially resolve previously identified candidate companions around WASP-12 and HAT-P-8 into two distinct sources. When combined with previous observations from Bergfors et al. (2013), our astrometric measurements show that WASP-12 BC and HAT-P-8 BC are gravitationally bound to one another as well as the primary, making WASP-12b and HAT-P-8b members of hierarchical triple star systems.

Our diffraction-limited measurements show that the two companions around WASP-12 are separated by $84.3 \pm 0.6$ mas ( $21 \pm 3 \mathrm{AU})$ and have roughly equal brightness. We estimate spectral types of M3V, consistent with the Crossfield et al. 2012 (spatially unresolved) combined-light spectroscopic analysis. Our photometric measurements combined with evolutionary models indicate masses of $0.38 \pm 0.05 M_{\odot}$ and $0.37 \pm 0.05 M_{\odot}$ for WASP-12 B and C, respectively. The companions orbiting HAT-P- 8 are separated by only $65.3 \pm 0.5$ mas $(15 \pm 1$ AU) and have somewhat more disparate properties. We estimate that HAT-P-8 B has a mass of $0.22 \pm 0.03 M_{\odot}$ and HAT-P-8 $\mathrm{C}$ has a mass of $0.18 \pm 0.02 M_{\odot}$. In each case our ability to characterize each system is limited by the lack of an accurate trigonometric parallax.

The ongoing debate concerning the origin of misaligned hot Jupiters has brought about several potential orbital evolutionary theories. AO imaging shows significant promise to improve our understanding of the dynamical history of these systems. Although numerous candidate companions around hot Jupiter hosts have been identified (e.g., Bergfors et al. 2013), multiepoch astrometry that assesses the physical association of these objects requires dedicated follow-up measurements from comprehensive programs that study close-separations stellar companions in detail. WASP-12 and HAT-P-8 may offer unique insights into the dynamics of hot Jupiter systems because their hierarchy will ultimately enable companion mass estimates using dynamics.

This research has made use of the SIMBAD database, operated at CDS, Strasbourg, France. Data presented herein were obtained at the W.M. Keck Observatory, which is operated as a scientific partnership among the California Institute of Technology, the University of California, and the National Aeronautics and Space Administration. The Observatory was made possible by the generous financial support of the W.M. Keck Foundation. J.A.J. is supported by generous grants from the David and Lucile Packard Foundation and the Alfred P. Sloan Foundation. B. T. M. is supported by the National Science Foundation Graduate Research Fellowship under Grant No. DGE1144469.

\section{REFERENCES}

Albrecht, S., Winn, J. N., Johnson, J. A., et al. 2012, ApJ, 757, 18 Batygin, K. 2012, Natur, 491, 418
Beaugé, C., \& Nesvorný, D. 2012, ApJ, 751, 119

Bergfors, C., Brandner, W., Daemgen, S., et al. 2013, MNRAS, 428,182

Bergfors, C., Brandner, W., Henning, T., \& Daemgen, S. 2011, in IAU Symp. 276, The Astrophysics of Planetary Systems: Formation, Structure, and Dynamical Evolution, ed. A. Sozzetti, M. G. Lattanzi, \& A. P. Boss (Cambridge: Cambridge Univ. Press), 397

Chatterjee, S., Ford, E. B., Matsumura, S., \& Rasio, F. A. 2008, ApJ, 686, 580

Crepp, J. R., Johnson, J. A., Howard, A. W., et al. 2012, ApJ, 761, 39

Crossfield, I. J. M., Barman, T., Hansen, B. M. S., Tanaka, I., \& Kodama, T. 2012, ApJ, 760, 140

Daemgen, S., Hormuth, F., Brandner, W., et al. 2009, A\&A, 498, 567

Dawson, R. I., \& Murray-Clay, R. A. 2013, ApJL, 767, L24

Dupuy, T. J., Liu, M. C., Bowler, B. P., et al. 2010, ApJ, 721, 1725

Eggenberger, A., Udry, S., Chauvin, G., et al. 2007, A\&A, 474, 273

Fabrycky, D. C., \& Winn, J. N. 2009, ApJ, 696, 1230

Faedi, F., Staley, T., Gómez Maqueo Chew, Y., et al. 2013, MNRAS, 433, 2097

Fleming, S. W., Ge, J., Barnes, R., et al. 2012, AJ, 144, 72

Ford, E. B., \& Rasio, F. A. 2008, ApJ, 686, 621

Fossati, L., Ayres, T. R., Haswell, C. A., et al. 2013, ApJL, 766, L20

Fossati, L., Haswell, C. A., Froning, C. S., et al. 2010, ApJL, 714, L222

Ghez, A. M., Salim, S., Weinberg, N. N., et al. 2008, ApJ, 689, 1044

Ginski, C., Mugrauer, M., Seeliger, M., \& Eisenbeiss, T. 2012, MNRAS, 421,2498

Girardi, L., Bertelli, G., Bressan, A., et al. 2002, A\&A, 391, 195

Goldreich, P., \& Tremaine, S. 1980, ApJ, 241, 425

Hebb, L., Collier-Cameron, A., Loeillet, B., et al. 2009, ApJ, 693, 1920

Hébrard, G., Ehrenreich, D., Bouchy, F., et al. 2011, A\&A, 527, L11

Høg, E., Fabricius, C., Makarov, V. V., et al. 2000, A\&A, 355, L27

Knutson, H. A., Fulton, B. J., Montet, B. T., et al. 2014, ApJ, 785, 126

Kraus, A. L., \& Hillenbrand, L. A. 2007, AJ, 134, 2340

Lai, D., Foucart, F., \& Lin, D. N. C. 2011, MNRAS, 412, 2790

Latham, D. W., Bakos, G. Á., Torres, G., et al. 2009, ApJ, 704, 1107

Li, S.-L., Miller, N., Lin, D. N. C., \& Fortney, J. J. 2010, Natur, 463, 1054

Lin, D. N. C., Bodenheimer, P., \& Richardson, D. C. 1996, Natur, 380, 606

Madhusudhan, N., Harrington, J., Stevenson, K. B., et al. 2011, Natur, 469, 64

Mancini, L., Southworth, J., Ciceri, S., et al. 2013, A\&A, 551, A11

Mason, B. D., Hartkopf, W. I., Raghavan, D., et al. 2011, AJ, 142, 176

Morton, T. D., \& Johnson, J. A. 2011, ApJ, 729, 138

Moses, J. I., Madhusudhan, N., Visscher, C., \& Freedman, R. S. 2013, ApJ, 763,25

Moutou, C., Díaz, R. F., Udry, S., et al. 2011, A\&A, 533, A113

Mugrauer, M., \& Neuhäuser, R. 2009, A\&A, 494, 373

Murray, N., Hansen, B., \& Holman, M. 1998, JRASC, 92, 310

Nagasawa, M., \& Ida, S. 2011, ApJ, 742, 72

Naoz, S., Farr, W. M., Lithwick, Y., Rasio, F. A., \& Teyssandier, J. 2011, Natur, 473,187

Narita, N., Takahashi, Y. H., Kuzuhara, M., et al. 2012, PASJ, 64, L7

Pollack, J. B., Hubickyj, O., Bodenheimer, P., et al. 1996, Icar, 124, 62

Roberts, L. C., Jr., Turner, N. H., ten Brummelaar, T. A., Mason, B. D., \& Hartkopf, W. I. 2011, AJ, 142, 175

Simpson, E. K., Pollacco, D., Cameron, A. C., et al. 2011, MNRAS, 414, 3023

Skrutskie, M. F., Cutri, R. M., Stiening, R., et al. 2006, AJ, 131, 1163

Stevenson, K. B., Bean, J. L., Seifahrt, A., et al. 2013, arXiv:1305.1670

Swain, M., Deroo, P., Tinetti, G., et al. 2013, Icar, 225, 432

Triaud, A. H. M. J., Collier Cameron, A., Queloz, D., et al. 2010, A\&A, 524, A25

Winn, J. N., Fabrycky, D., Albrecht, S., \& Johnson, J. A. 2010a, ApJL, 718, L145

Winn, J. N., Johnson, J. A., Albrecht, S., et al. 2009, ApJL, 703, L99

Winn, J. N., Johnson, J. A., Howard, A. W., et al. 2010b, ApJ, 718, 575

Wizinowich, P, Acton, D. S., Shelton, C., et al. 2000, PASP, 112, 315

Wu, Y., \& Murray, N. 2003, ApJ, 589, 605

Yelda, S., Lu, J. R., Ghez, A. M., et al. 2010, ApJ, 725, 331

Zimmerman, N., Oppenheimer, B. R., Hinkley, S., et al. 2010, ApJ, 709, 733 